\title{
LA CONSERVACIÓN DEL PATRIMONIO PALEONTOLÓGICO EN ESPAÑA: EL PAPEL DE LA ADMINISTRACIÓN Y DE LOS PALEONTÓLOGOS
}

\author{
Guillermo MELÉNDEZ, Celia SORIA-LLOP \\ y Montserrat SORIA VERDE
}

Dpto. de Geología (Paleontología). Universidad de Zaragoza. c./Pedro

Cerbuna, 12. 50009 Zaragoza. e-mail: gmelende@posta.unizar.es

Meléndez, G., Soria-Llop, C. y Soria Verde, M. 2001. La conservación del patrimonio paleontológico en España: el papel de la Administración y de los paleontólogos. [Preserving the palaeontological heritage in Spain: The role played by the Administration and palaeontologists.] Revista Española de Paleontología, no extraordinario, 8598. ISSN 0213-6937.

\begin{abstract}
The palaeontological heritage is formed, on one side, by fossil sites and fossiliferous sections studied by the palaeontological communnity (the so-called immobile heritage). On the other side, by public and private palaeontological collections and specimens, stored in palaeontological museums, universities, research institutions and private owners (the so-called mobile heritage). Main threats on palaeontological heritage are the obvious risks to any geological site, such as destruction by public works, but also the destruction and looting by amateur collectors and traders. This has led to a scenario of serious risk as regards its existence and conservation, a situation the palaeontological communnity has only recently been aware.

The development, between 1985 and 1989 of a national legislation on historical and natural, including palaeontological heritage, has considerably favoured the regulation and instauration of protective actions. Yet these first national laws have been subsequently expanded differently in the Autonomous Communnities by the regional governments of Spain. Legal development has been generally favourable for palaeontological heritage although it has not always been satisfactory for professional palaeontologists: Restrictive regulations on palaeontological excavations are effective in most of spanish regions. Yet only in few of these protective measures, such as the legal definition of an important point as a protected site, or the development of active policy of social and educational management of fossil sites, are being actively promoted. This is, anyway, the beginning of a road that should progress towards the nomination of most important fossil sites as protected points, according to the existant heritage laws.

The Spanish Palaeontological Society (SEP) by means of the Heritage Commission, should play a relevant role in this process, as an intermediate partner between palaeontologists and the Administration, but also as the "specialists" assembly" carrying on the fundamental task of defining, characterising and categorising fossil sites by proposing the creation of specifical features to remark the importance of a site by its scientific or heritage value. The proposal by the Heritage Commission of creating a palaeontological non-formal, internal feature; the so-called Point of Special Palaeontological Interest (PEIP) might surely help progress in this long-term purpose of defining and protecting the most important palaeontological sites.
\end{abstract}

Keywords: Palaeontological heritage, heritage laws, Spain, Autonomous Communnities, Spanish Palaeontological Society, Heritage Commission, Point of Special Palaeontological Interest (PEIP).

\section{RESUMEN}

El patrimonio paleontológico está constituido, por un lado, por el conjunto de yacimientos y secciones fosilíteras conocidos y estudiados (patrimonio "inmueble") y por otro, por el conjunto de colecciones paleontológicas y ejemplares alojados en museos, colecciones y exposiciones locales (patrimonio "mueble"). Los riesgos que afectan al patrimonio paleontológico son fundamentalmente el de destrucción y el de expolio por aficionados y comerciantes. Todo ello ha generado una situación de riesgo evidente para su conservación de la que la comunidad paleontológica española sólo ha comenzado a tomar conciencia en los tiempos recientes.

El surgimiento en 1985, y posteriormente en 1989, de una legislación nacional sobre patrimonio y sobre espacios naturales, desarrollada de manera diversa en las diferentes Comunidades Autónomas del Estado 
Español, ha venido a incidir en esta problemática de manera favorable aunque no siempre satisfactoria para todas las partes. En bastantes comunidades autónomas las regulaciones sobre excavaciones paleontológicas se encuentran efectivas desde hace tiempo y en algunas se inician políticas activas destinadas a la protección y/o a la gestión y difusión social de los yacimientos. Se inicia así un proceso que deberá progresar hacia la declaración de los yacimientos de mayor importancia como puntos protegidos de acuerdo con las figuras legales existentes.

La Sociedad Española de Paleontología, por medio de la Comisión de Patrimonio, tiene una importante labor que realizar en este campo, como intermediario entre los paleontólogos y la Administración, pero también la tarea fundamental de definir, caracterizar, sistematizar y jerarquizar los yacimientos paleontológicos según su valor patrimonial y su importancia, mediante la creación de figuras específicas que señalen el interés y el grado de protección de un yacimiento o de una sección por su valor científico y patrimonial. En este sentido, la propuesta de creación de la figura interna, sin status legal, de Punto de Especial Interés Paleontológico (PEIP) constituye un paso que contribuirá a avanzar en el proceso de definir y proteger legalmente el patrimonio paleontológico.

\section{Palabras clave: Patrimonio paleontológico, legislación, España, Comunidades Autónomas, Sociedad Española de Paleontología, Comisión de Patrimonio, Punto de Especial Interés Paleontológico (PEIP).}

\section{INTRODUCCIÓN}

En un sentido general, el patrimonio paleontológico de un país o de una región puede entenderse como constituido por el registro fósil, es decir: el conjunto de entidades registradas, existentes en dicho país, de acuerdo con la definición original de entidad registrada (Fernández-López, 1981). En una visión más actualizada y restringida, sin embargo, el patrimonio paleontológico lo forman por un lado el conjunto de yacimientos conocidos y estudiados por la comunidad paleontológica (patrimonio inmueble) y por otro, el conjunto de colecciones, de ejemplares, museos y exposiciones que conforman el material utilizado en investigación, así como para fines didácticos o de difusión social de la paleontología (patrimonio mueble). Esta definición coincidiría por tanto con el concepto de entidad obtenida y estimada del mismo autor (Fernández-López, op. cit.). Aunque complementarios e interdependientes, ambos tipos de patrimonio, el inmueble y el mueble, tienen distintas características y requerimientos y pueden ser definidos y analizados de modo diferente. Mientras que el hallazgo, explotación, clasificación y estudio del patrimonio corresponde a la comunidad paleontológica, su gestión, definición legal, difusión social y utilización didáctica corresponde a las entidades sociales y, en último término, a la autoridad política. Entendido el patrimonio de esta manera como un bien social, la comunidad paleontológica se erige como depositaria temporal y responsable de la conservación dicho patrimonio mientras que la sociedad, por medio de sus representantes, se responsabiliza de su gestión.

El surgimiento de una legislación que afecta a, y regula la gestión del patrimonio paleontológico (Ley 6/85, del Patrimonio Histórico Español, de 1985 y Ley 4/89, de Conservación de los Espacios Naturales y de la Flora y Fauna Silvestres, de 1989) ha influido en el desarrollo de la investigación paleontológica en nuestro país y ha generado no pocos conflictos entre los paleontólogos y la Administración (cf. López Martínez, 1995; Sanz et al., 1995). No obstante, también ha influido en alguna medida positivamente sobre la comunidad paleontológica y sobre la sociedad en general, al promover un debate y contribuir a crear una conciencia sobre la necesidad de proteger el patrimonio paleontológico y favorecer su gestión y difusión social (Alcalá y Paricio, 1984, 1988; Alcalá, 1992; Grupo de Trabajo sobre Protección de Yacimientos, 1993).

En el seno de la Sociedad Española de Paleontología (SEP) la Comisión de Patrimonio, creada en 1997, se ha constituido con la intención de cumplir esa doble función: Por un lado, servir de "puente" entre la Sociedad y la Administración ejerciendo tanto una labor informativa en el interior de la Sociedad, hacia los paleontólogos, como asesora o de ayuda e información hacia la Administración si ésta lo requiere. Por otro lado, contribuir a la definición y sistematización del patrimonio elaborando un banco de datos de los puntos paleontológicos de mayor interés (Meléndez et cil., 1999).

El objetivo del presente trabajo es mostrar la situación actual del patrimonio paleontológico y el desarrollo de todos los puntos en el seno de la comunidad paleontológica española así como la labor realizada por la Comisión de Patrimonio desde su constitución como órgano delegado de la SEP.

\section{RIESGOS Y DETERIORO DEL PATRIMONIO}

Los riesgos a que puede verse sometido el patrimonio paleontológico son múltiples, derivados de sus propias características intrínsecas (ubicación, accesibilidad, riqueza de los yacimientos y posibilidades de destrucción y erosión) así como de su interés como bien social, económico y comercial. Con respecto al primer punto, la ubicación y accesibilidad de un yacimiento paleontológico influye y condiciona su importancia puesto que existe una relación directa entre la accesibilidad de un yacimiento y su facilidad de estudio. De tal manera, no es de extrañar que los yacimientos más accesibles y conocidos sean además los más estudiados y los que han sido escogidos preferentemente como puntos de referencia (estratotipos, cortes de referencia, localidades tipo de unidades estratigráficas). Esta accesibilidad puede, no obstante, jugar también en su contra al convertirse preferentemente en punto de afección por obras públicas, ensanche de carreteras, 
ubicación de canteras o de vertederos públicos, y en objeto más directo de expolio (Meléndez y Soria, 1999).

\section{TIPIFICACIÓN DEL EXPOLIO}

Los riesgos de expolio y destrucción vienen derivados de la tradición antigua (que se remontaría de hecho a los orígenes de las civilizaciones humanas) del coleccionismo de fósiles por el hombre, por su atractivo como objetos curiosos o como piezas decorativas o artísticas (Page et al., 1999). En la actualidad son los paleontólogos aficionados, los excursionistas, coleccionistas ocasionales y comerciantes y expoliadores profesionales los que ocasionan un mayor daño al patrimonio paleontológico. La tipología del coleccionistaexpoliador, ocasional, aficionado o profesional es amplia y se encuentra enormemente extendida en muchos países. Sobre sus efectos, beneficiosos o perjudiciales para la paleontología, se ha debatido en profundidad en numerosos foros y el debate se promete largo.

En la actualidad el patrimonio paleontológico se encuentra contemplado en el marco legal dentro de nuestro país, y la explotación de yacimientos y las excavaciones se encuentran reguladas por ley en las distintas comunidades autónomas. De acuerdo con esto la recolección no autorizada de fósiles constituye una acción fuera de la ley. En este punto es necesario destacar sin embargo que muchas acciones de "expolio" o de muestreo incontrolado o realizado con fines didácticos o sociales (el maestro que lleva a los alumnos a recoger fósiles al campo) pueden tener una incidencia mínima sobre los yacimientos. Existe así una amplia banda de transición entre una acción aislada sin impacto alguno sobre el patrimonio (el hecho de recoger fósiles rodados del suelo en un campo) y la excavación ilegal y destrucción sistemática de un yacimiento (el verdadero expolio), realizado por profesionales comerciantes o coleccionistas. En el momento actual la ley no distingue entre unas acciones y otras, por lo que se da la situación poco razonable de que cualquier persona que recoja fósiles del suelo en el campo, sin estar en posesión de un permiso específico de la Administración, puede encontrarse fuera de la ley (Meléndez y Soria-Llop, 2000). Todo esto configura un amplio campo temático por debatir y bastantes aspectos por definir y delimitar en lo referente al uso, gestión y utilización del patrimonio (Alcalá y Morales, 1994).

\section{CARACTERIZACIÓN DEL PATRIMONIO PALEONTOLÓGICO}

Resulta por tanto de primordial interés para la comunidad paleontológica y para la sociedad, establecer de modo preciso y consensuado el significado real de muchos conceptos sobre patrimonio, algunos ya definidos pero escasamente demarcados. Entre ellos, el concepto mismo de patrimonio, de yacimiento paleontológico, de nivel fosilífero, de ejemplar de interés, etc., así como
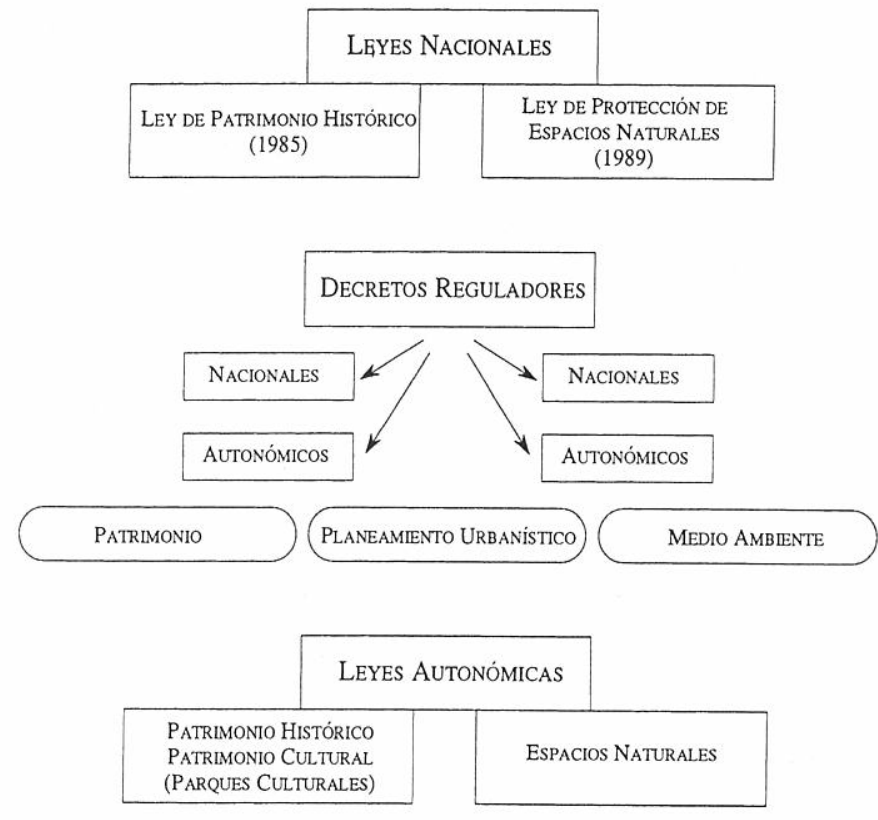

Figura 1. Esquema del desarrollo del marco legislativo sobre patrimonio en España, en la parte que ha afectado al patrimonio paleontológico, desde la aparición de las leyes nacionales de Patrimonio Histórico (1985) y de Protección de Espacios Naturales (1989).

Development of the general legal framework on historical heritage in Spain, with special reference to palaeontology. First national-scale laws are the Historical Heritage Law (1985) and the Law on Protection of Natural Areas (1989).

establecer una jerarquía de criterios sobre los aspectos que confieren un mayor o menor interés a un yacimiento paleontológico. En un trabajo reciente, J. Morales (1996) realiza una interesante ordenación de los distintos tipos de criterios que pueden contribuir a definir el mayor o menor interés de los yacimientos. El autor distingue varios tipos de criterios:

- Criterios científicos, resultantes de las características propias del yacimiento.

- Criterios socio-culturales, relativos a su conservación, uso y gestión.

- Criterios socio-económicos, relacionados con el entorno político-social, y con circunstancias generalmente ajenas a los propios yacimientos. De acuerdo con el autor, estos criterios "suelen imponer limitaciones al conjunto patrimonial”.

El debate, y el deseable consenso sobre estos aspectos, promovido en el seno de la Sociedad Española de Paleontología y en coordinación con las administraciones públicas, debería servir de base para seleccionar los yacimientos paleontológicos que deban ser propuestos como puntos protegidos. En este sentido, las relaciones entre el desarrollo del marco legal y los riesgos y las acciones sobre la protección del patrimonio paleontológico han sido objeto de numerosos análisis ( $c f$. Alcalá y Paricio, 1984; 1988; Soria y Meléndez, 1993). 


\begin{tabular}{|l|l|l|l|l|l|}
\hline $\begin{array}{c}\text { Comunidad } \\
\text { Autónoma }\end{array}$ & Ley de patrimonio & $\begin{array}{c}\text { Otras Normativas } \\
\text { (excavaciones) }\end{array}$ & $\begin{array}{c}\text { Figura de protección } \\
\text { Paleontología }\end{array}$ & $\begin{array}{l}\text { Ley de Espacios } \\
\text { Naturales }\end{array}$ & $\begin{array}{c}\text { Figura de protección } \\
\text { Paleontología/Geología }\end{array}$ \\
\hline Andalucia & Ley 1/1991 & Decreto 32/1993 & No tiene & Ley 2/1989 & PN, MN, PjN \\
\hline Aragón & $\begin{array}{l}\text { Ley 12/1997 Parq C. } \\
\text { Ley 3/1999 PCA }\end{array}$ & Decreto 6/1990 & Zona Paleontológica & Ley 6/1998 & P, MN, PN, ENP \\
\hline Asturias & Prop. 11/01/1999 & Decreto 64/1986 & Zona Paleontológica & Ley 5/1991 & PN, MN \\
\hline Baleares & Ley 12/1998 & Orden 10/05/1986 & Zona Paleontológica & $\begin{array}{c}\text { Ley 1/1984 } \\
\text { Ley 1/1991 }\end{array}$ & PN, MN, AEP \\
\hline Canarias & Ley 4/1999 (PHC) & & SH, ZA (Ley 16/1985) & Ley 12/1994 & PN, MN \\
\hline Cantabria & Ley 11/1998 & Decreto 72/1985 & LN (No tiene) & & P, MN (Ley 4/1989) \\
\hline Castilla-León & & Decreto 37/1985 & SH, ZA (Ley 16/1985) & Ley 8/1991 & P, MN \\
\hline C. La Mancha & Ley 4/1990 & & SH, ZA & & P, MN (Ley 4/1989) \\
\hline Cataluña & Ley 9/1993 & Decreto 231/1991 & Zona Paleontológica & Ley 12/1985 & Reserva Natural Parcial \\
\hline Extremadura & & Decreto 93/1997 & SH, ZA (Ley 16/1985) & & P, MN (Ley 4/1989) \\
\hline Galicia & & Decreto 199/1997 & SH, ZA (Ley 16/1985) & & P, MN (Ley 4/1989) \\
\hline Madrid & Ley 10/1998 & Orden 24 /06/1986 & Zona Paleontológica & & P, MN (Ley 4/1989) \\
\hline Murcia & & Decreto 180/1987 & SH, ZA (Ley 16/1985) & Ley 4/1992 & P, MN (Ley 4/1989) \\
\hline Navarra & & Decreto 218/1986 & SH, ZA (Ley 16/1985) & $\begin{array}{l}\text { Ley 6/1987 } \\
\text { Ley 2/1993 }\end{array}$ & PN, MN, RN (Ley 4/89) \\
\hline Pais Vasco & Ley 7/1990 & & CM (ZA/PA) & Ley 16/1994 & Biotopo protegido \\
\hline La Rioja & & & SH, ZA (Ley 16/1985) & & P, MN (Ley 4/1989) \\
\hline Valencia & Ley 4/1998 & Orden 31/07/1987 & Zona Paleontológica & & P, MN (Ley 4/1989) \\
\hline
\end{tabular}

Figura 2. Desarrollo del marco legal sobre patrimonio paleontológico en España, en las comunidades autónomas, actualizado hasta el año 1999 (ver también las sucesivas versiones anteriores en Meléndez y Soria, 1997, 1999). SH: Sitio Histórico; ZA: Zona Arqueológica; LN: Lugar Natural; CM: Conjunto Monumental; PA: Parque Arqueológico; PN: Parque Natural; MN: Monumento Natural; PjN: Paraje Natural; P: Parque; ENP: Espacio Natural Protegido; AEP: Area de Especial Protección; RN: Reserva Natural.

Development of the legal framework on palaeontological heritage in the different regions ("Authonomous Communnities") of Spain, updated to 1999 (see the successive versions published in Meléndez and Soria, 1997, 1999). SH: Historical Site; ZA: Archaeological Zone; LN: Natural Area; CM: Monumental Assemblage; PA: Archaeological Park; PN: Natural Park; MN: Natural Monument; PjN: Natural Landscape; P: Park; ENP: Protected Natural Area; AEP: Special Protection Area; RN: Natural Reserve.

\section{MEDIDAS DE PROTECCIÓN}

Las medidas tendentes a proteger el patrimonio y evitar la destrucción de yacimientos son de muy diversa índole y dependen en gran medida del tipo concreto de riesgos que puedan afectar a aquéllos. No obstante, hay que señalar que todas la medidas externas a la comunidad científica pueden agruparse en dos categorías: políticas, y educativas o didácticas.

\section{MEDIDAS POLÍTICAS}

Las medidas políticas comprenden la aplicación de los recursos contemplados en la legislación vigente para declarar un yacimiento como área protegida por medio de su definición como una figura específica dentro de las dos leyes fundamentales de alcance nacional (Fig. 1). Estas son:

- La Ley 16/1985, de 25 de Junio, de Patrimonio
Histórico Español. Esta ley define la figura fundamental de Bien de Interés Cultural (B.I.C.) como instrumento legal para proteger un valor patrimonial, entre ellos los yacimientos paleontológicos. Estos pueden ser declarados B.I.C. bajo las dos acepciones posibles de Sitio Histórico y de Zona Arqueológica.

- La Ley 4/1989, de 27 de Marzo de Conservación de los Espacios Naturales y de la Flora y Fauna Silvestres. Esta ley define la figura fundamental de Parque Natural, como un espacio protegido en el cual pueden incluirse los yacimientos paleontológicos. En concreto, un yacimiento paleontológico o un rasgo geológico significativo pueden ser declarados con la denominación de Monumento Natural.

Estas leyes de ámbito estatal se han visto complementadas, a nivel nacional o bien a nivel autonómico, por un conjunto de reales decretos o decretos reguladores que abarcan otros aspectos de detalle del patrimonio. Asimismo, algunas comunidades 


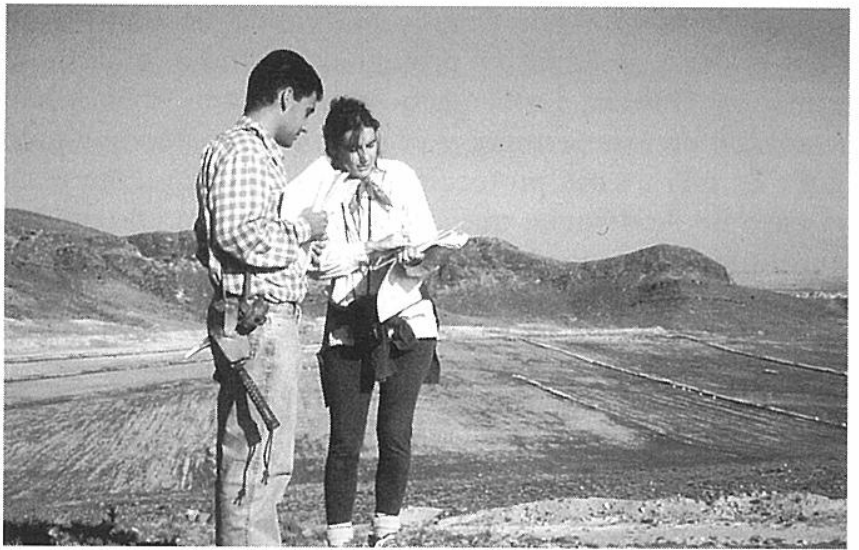

Figura 3. Trabajos de prospección previa para la realización de obras públicas: durante el informe previo al trazado de la línea férrea del tren de alta velocidad (AVE) Madrid-Zaragoza, en Ricla (Zaragoza), afloramiento Ri.5 (1995).

Palaeontological prospective work for public works in Aragón. Field work corresponding to the previous impact report for the high-speed railway line Madrid - Zaragoza in the region of Ricla (Zaragoza; outcrop Ri.5; 1995).

autónomas ya cuentan con las correspondientes leyes propias, regionales, de Patrimonio Cultural, o Patrimonio Histórico, y de Medio Ambiente, o de Protección de Espacios Naturales, habiendo definido en algunos casos una figura específicamente paleontológica de protección (Fig. 2). Este es el caso de las comunidades autónomas de Aragón, Baleares, Cataluña, Madrid y Valencia, que, en la Ley Autonómica de Patrimonio cuentan con la figura específica de Zona Paleontológica. Esta figura ha venido a salvar en cierta medida el histórico "desequilibrio" legal de la legislación sobre Patrimonio en favor de la Arqueología existente en la Ley Nacional de Patrimonio Histórico, al contemplar únicamente las denominaciones de Sitio Histórico y de Zona Arqueológica dentro de la figura legal de Bien de Interés Cultural. Una síntesis relativamente actualizada de las disposiciones legales sobre este punto, a nivel estatal y más concretamente en la Comunidad Autónoma de Aragón, se encuentra en Andrés-Moreno y Royo-Guillén (1998). En conjunto, las normativas más directamente ligadas al patrimonio paleontológico son:

- El Real Decreto 111/1986 de 10 de Enero de desarrollo parcial de la Ley 16/1985 de Patrimonio Histórico Español, que regula muchos aspectos, como la tramitación de la declaración de Bien de Interés Cultural (B.I.C.), y los aspectos relativos al patrimonio mueble.

- El Real Decreto 6/1990 de 23 de Enero del Gobierno de Aragón, que regula las prospecciones y excavaciones paleontológicas. Esta regulación se ha desarrollado en las distintas comunidades autónomas por medio de decretos semejantes, tal como puede verse en el cuadro legislativo actualizado de la Fig. 2.

Otras medidas políticas son la exigencia de realizar informes previos en los proyectos de grandes obras públicas sobre impacto ambiental, dentro de los cuales se

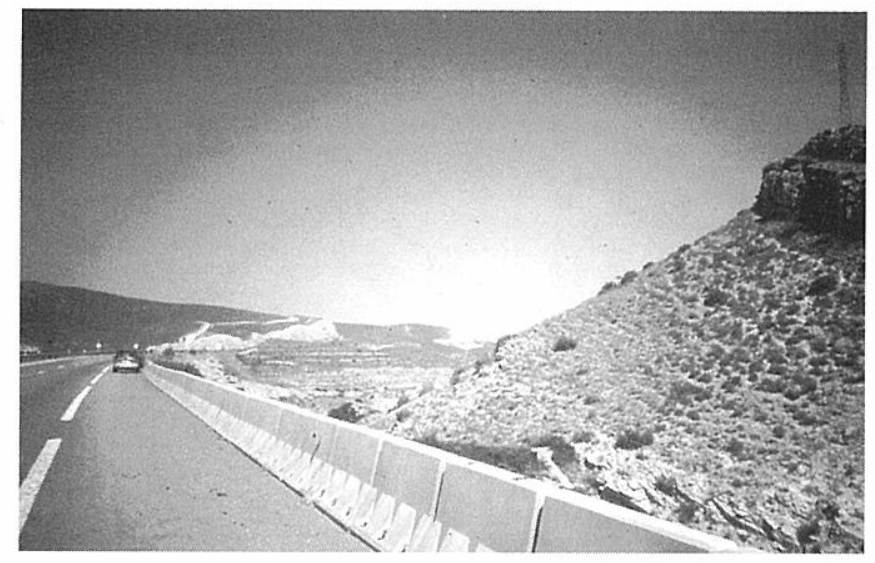

Figura 4. Vista parcial de la Autovía de Aragón (A-2) a su paso por La Almunia de Doña Godina (Zaragoza), al cruzar los afloramientos jurásicos de la Rama Aragonesa de la Cordillera Ibérica. En este punto, el trazado previo de la autovía afectaba gravemente a la sección del Toarciense ("Camino de las Conchas"), propuesta como sección de referencia para Europa occidental. El informe sobre impacto paleontológico consiguió que el trazado se modificara ligeramente para evitar su destrucción o su desaparición al ser cubierta por el talud de la autovía.

Partial view of the Aragón Motorway (A-2) in the locality of La Almunia de Doña Godina (Zaragoza), crossing through the Jurassic outcrops of the Aragonese Branch of Iberian Cordillera. A prior design of the road would seriously affect a traditional outcrop of the Toarcian fossiliferous beds known as "the shell road", which had been proposed as reference section for this interval in West-Europe. A palaeontological impact report succeeded in slightly modifying the original design, hence avoiding the destruction of the outcrop or its burial by slope debris.

incluye el impacto paleontológico, tal como recoge el Real Decreto Legislativo 1302/1986 de 28 de Junio sobre Evaluación de Impacto Ambiental, y a nivel autonómico en el Decreto 45/1994, de 4 de Marzo, de la Comunidad Autónoma de Aragón sobre Evaluación de Impacto Ambiental (Fig. 3). De igual manera, el descubrimiento de un importante yacimiento durante una prospección correspondiente a uno de estos informes en un punto que se verá afectado por una obra pública, puede dar lugar a una modificación de aquélla, como ha sido el caso de la Autovía de Aragón a su paso por La Almunia de Doña Godina (Fig. 4) o al menos a la realización de una excavación de urgencia con objeto de extraer una parte del material paleontológico que luego se verá afectado por la remoción de terrenos. Casos como éste han sido relativamente frecuentes durante la prospección y realización de las obras del tendido del tren de alta velocidad (AVE) en España, y concretamente en las obras del trazado de la línea Madrid-Barcelona, tal como se muestra en la Fig. 5. 


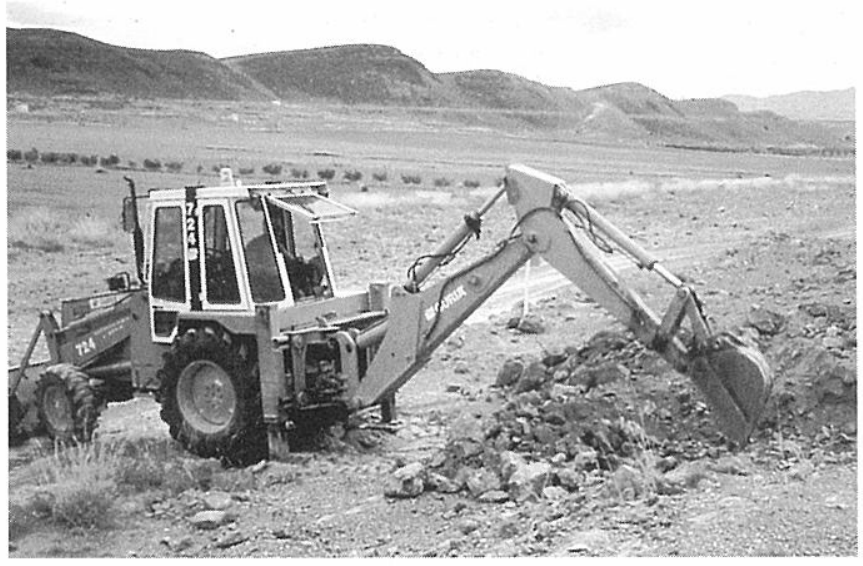

Figura 5. Excavación de una zanja en las obras de construcción del trazado de la línea del tren de alta velocidad (AVE) a su paso por Ricla (Zaragoza; afloramiento Ri.5). Esta zanja, abierta en los materiales del Calloviense-Oxfordiense (Jurásico Medio-Superior) permitió realizar una excavación de urgencia en estos materiales, en el punto de cruce por el trazado de la vía férrea, tras haber sido señalado en el informe paleontológico previo (v. Fig. 3).

"Rescue dig" in the Middle-Upper Jurassic deposits (Callovian-Oxfordian) near Ricla (Zaragoza; outcrop Ri.5) involving the excavation of a trench. The projected design of the high-speed train (AVE) line MadridZaragoza, meant a serious threaten to jurassic outcrops in this area. However, as proposed in the conclusions of the palaeontological impact report, this rescue dig, kindly awarded and financed by the public works company, allowed the stratigraphic study and palaeontological sampling of the section before being covered by the railway slope (see Fig. 3).

Es indudable que las medidas derivadas de la aplicación de las leyes de Patrimonio y de Espacios Naturales en las diferentes comunidades autónomas, por ejemplo, la regulación de las prospecciones y excavaciones paleontológicas, la declaración de yacimientos como puntos de especial interés (o su inclusión dentro de áreas protegidas) y la obligación de realizar informes de impacto ambiental, en el que se incluye el impacto paleontológico, en las distintas obras públicas, está siendo beneficioso para el patrimonio paleontológico y deberá serlo aún más en un futuro. No obstante, es preciso tener en cuenta los siguientes puntos:

\section{PROBLEMÁTICA DE LAS MEDIDAS POLÍTICAS}

\section{Desarrollo autonómico desigual}

Aun cuando las dos leyes básicas que afectan al patrimonio son de alcance nacional, su desarrollo legislativo ha sido bastante desigual en las distintas comunidades autónomas. Así, mientras algunas destacan por haber desarrollado una política activa de protección de yacimientos y de regulación de excavaciones, creando figuras legales específicas y nuevas leyes locales de Patrimonio o de Espacios protegidos (como es el caso de Cataluña, Galicia, La Rioja o Aragón) otras apenas han avanzado en este sentido, y el patrimonio paleontológico goza de una escasa protección activa (Fig. 2). Este sería el caso de Andalucía, que, siendo la primera comunidad autónoma en crear su propia ley de patrimonio (Ley 1/1991), en ella no aparece definida una figura paleontológica específica.

\section{Permisos de prospección y de excavación}

Una vez que las excavaciones y prospecciones paleontológicas (así como el destino posterior del material paleontológico) se encuentran reguladas por ley, los paleontólogos se encuentran obligados a seguir una serie de trámites burocráticos que en muchas ocasiones vienen a entorpecer o a dificultar de manera considerable su labor investigadora. Una vez culminada la penosa labor de solicitar y conseguir, con mayor o menor éxito, un proyecto de investigación a un organismo público (para un período de tres o cuatro años) deben comenzar un no menos tortuoso proceso de solicitud de permisos a las administraciones locales y autonómicas (Meléndez y Soria, 1999). Los permisos deben ser renovados anualmente y en ellos muchos detalles solicitados escapan a la programación de dichos proyectos (por ejemplo, el calendario detallado, la localización precisa de los puntos a prospectar, etc.) puesto que muchos de estos apartados dependen en parte del propio desarrollo del proyecto.

\section{Depósito del material estudiado}

Los problemas surgidos en este apartado son múltiples y no siempre fáciles de abordar. En este punto confluyen (en ocasiones se enfrentan) la legítima propiedad del material (la administración que ha otorgado el permiso y que deberá disponer en dónde debe ser depositado el material) y la preocupación por parte del autor de la recolección, preparación y estudio del material (el investigador) de que un material científico, cuya obtención y estudio ha costado años de trabajo y un cuantioso presupuesto, sea alojado y depositado en condiciones no siempre óptimas. En este sentido, las condiciones fundamentales que debe cumplir una colección tras su estudio son las siguientes:

- Seguridad: La colección debe ser almacenada en condiciones rigurosas de orden y seguridad, a salvo tanto de accidentes e imprevistos como de los problemas derivados de eventuales remodelaciones, traslados, o cambios en los criterios o programas políticos. Obviamente, sólo las instituciones públicas, museísticas o universitarias, y algunas fundaciones o colecciones privadas, pueden garantizar este requisito, y tampoco permanentemente. Con más frecuencia de la deseable, un material paleontológico puede hallarse expuesto a futuros e imprevisibles cambios por las autoridades (traslados, falta de espacio, almacenamiento incorrecto, reformas en los programas museísticos, etc.) pudiendo en ocasiones perderse un material de valor científico incalculable $y$ dejar de estar accesible y contrastable una información ya 
publicada. Todo esto crea las naturales suspicacias entre la comunidad paleontológica y las administraciones y ha generado no pocos conflictos en los últimos años. Esta condición requiere por tanto un compromiso serio y a largo plazo de la autoridad política para garantizar la estabilidad del material, así como un esfuerzo de dotación presupuestaria en equipamiento e instalaciones adecuadas.

- Accesibilidad: La condición de accesibilidad es fundamental para el material paleontológico procedente de colecciones de investigación, por lo que supone de capacidad de continuación del trabajo por parte del propio investigador y de posibilidades de contrastación de los resultados por parte de otros investigadores. Resulta lógico pensar que la mayor accesibilidad de las colecciones para los investigadores se encontrará cuando éstas se alojen en su mismo centro de investigación. En muchos casos es fácil comprender que un material depositado tras su estudio en los fondos de un museo o un centro de la administración alejado de, o poco conectado temáticamente con, la investigación realizada, puede no encontrar allí las mejores condiciones de alojamiento. Esto ha favorecido tradicionalmente la tendencia a enriquecer las colecciones de los centros, tanto departamentos universitarios como museos, con el material procedente de los propios investigadores.

No obstante, es preciso tener en cuenta que, al igual que en la primera de las condiciones, la accesibilidad del material también requiere un nivel básico de instalaciones y equipamiento que no siempre se encuentra en condiciones óptimas en cualquier centro. La condición de accesibilidad, por tanto, debería promover o generar una reflexión seria, tanto en la Administración como entre los investigadores, sobre el destino más apropiado del material paleontológico tras su estudio. Y podemos apuntar además que dicha reflexión debería llevar preferentemente hacia soluciones "conservadoras", favoreciendo en lo posible la permanencia del material en los centros de investigación, con los investigadores responsables, obviamente los más interesados en que dicho material se encuentre en condiciones óptimas.

Esta iniciativa puede, ciertamente, generar problemas o entrar en conflicto con las directrices políticas de las instituciones autonómicas, plenas depositarias de la responsabilidad de la gestión del patrimonio, especialmente cuando el equipo de investigación pertenece a una comunidad autónoma diferente y los investigadores solicitan retener el material en un centro distante de dicha Comunidad. Este problema está surgiendo constantemente desde el momento en que los estudios paleontológicos no se encuentran, o no deberían encontrarse, condicionados por los límites geográficos.

Sin embargo, el problema más importante en este sentido es heredado: desde los inicios de los estudios paleontológicos en España, la fuerte centralización política y académica por un lado y la precariedad de medios e instalaciones adecuadas por otro, en provincias con un patrimonio paleontológico excepcional (Teruel, Guadalajara, Cuenca o Lérida) ha hecho que gran parte del material de muchos estudios y colecciones se haya alojado en museos y centros universitarios de Madrid y Barcelona, y más recientemente, en Zaragoza, como queda patente en el reciente estudio realizado sobre la provincia de Teruel (Soria, 1993). Estos hechos podrían llevar en teoría (y están llevando en la práctica en algunos casos) a las autoridades políticas responsables del patrimonio en los gobiernos autónomos a actitudes y situaciones poco satisfactorias para los investigadores al reclamar o simplemente dictaminar, el traslado o el depósito a corto plazo del material extraído o estudiado recientemente, en un centro distante o escasamente vinculado con los estudios paleontológicos (como puede ser, por ejemplo, un museo arqueológico o etnológico).

Todo ello ha generado, y previsiblemente seguirá generando tensiones entre los investigadores y la Administración cuando la realidad es que la relación entre ambas partes debería ser de colaboración estrecha. No obstante, también es cierto que se pueden encontrar soluciones imaginativas que permitan armonizar ambas posiciones, reconociendo (a) la legítima autoridad de la comunidad científica sobre el material extraído y estudiado, y su derecho a poder acceder a él en las condiciones antes indicadas y (b) la propiedad legal de la Administración sobre dicho material y su responsabilidad sobre su protección, gestión y conservación:

- Por un lado, la figura del depósito en sus diversas variantes: temporal, renovable o permanente, y el seguimiento y control periódico del material. Esta figura, con los medios y las facilidades actuales, es perfectamente realizable.

- Por otro lado, la posibilidad de selección del material que deba ser depositado periódicamente, distinguiendo el material museístico, de características más excepcionales y en cierto modo más emblemático, del material menos espectacular, que normalmente forma la parte más importante de las colecciones y que suele constituir la base de datos fundamental de cualquier trabajo. Las primeras piezas pueden ser reclamadas, y deben ser reintegradas en primer lugar, formando también la parte más llamativa, o socialmente más apreciada de los resultados de la investigación. Tal como ya afirman Morales et al. (en prensa), sólo una pequeña parte del material estudiado suele ser museístico o sujeto de exposición. Y éste puede encontrarse expuesto y perfectamente accesible en su legítimo lugar de destino. El resto del material, crucial para realizar nuevos trabajos pero poco o nada interesante para museos o centros ajenos al investigador, puede permanecer, convenientemente controlado y catalogado, por más tiempo, incluso indefinidamente, con el investigador.

- En tercer lugar, la necesidad de contar, a la hora de decidir el destino del material, con la opinión del investigador, teniendo en cuenta que constituye la opinión autorizada del especialista que, en la mayoría de los casos, ha descubierto, recolectado, preparado, estudiado y publicado el material en cuestión. Nada resulta más desmoralizador para un investigador que el recibir un escrito de una autoridad de la administración indicándole (en algunos casos conminándole) que debe depositar el material estudiado, y en ocasiones antes de 
haberlo estudiado, en un centro o en un museo ajeno a las actividades paleontológicas y en condiciones dudosas, o al menos desconocidas, de seguridad y accesibilidad. Así, la Administración no debe ignorar la autoridad del investigador sobre este punto; antes bien, debe buscar su colaboración en la medida de lo posible.

- Catalogación: Si las dos condiciones analizadas constituyen propiedades primordiales de las colecciones paleontológicas, esta tercera puede calificarse de fundamental, en cierta medida derivada de las otras dos y catalizadora de ellas: es fácil comprender que sólo cuando una colección se encuentre bien alojada, en condiciones de perfecta estabilidad, seguridad y accesibilidad, podrá estar bien catalogada. Pero no es menos cierto que una correcta catalogación de aquélla contribuirá a incrementar su seguridad y accesibilidad o facilidad de localización, y de seguimiento de ejemplares.

Los modernos programas de informatización de colecciones y las bases de datos desarrolladas en numerosos centros museísticos y universitarios en ciudades como Madrid, Barcelona, Valencia, Zaragoza, Oviedo o Salamanca resultan encomiables y modélicos en su empeño y sus resultados, y no cabe duda de que han dado pasos de gigante en el proceso de ordenación y catalogación de las colecciones paleontológicas. No obstante, las colecciones a inventariar son muchas y en continuo aumento, y el trabajo por realizar es inmenso si se quiere tener a disposición de los investigadores el material publicado, figurado o simplemente existente en colecciones de investigación y de comparación.

Los objetivos a cubrir en un futuro inmediato en este campo pasan por:

- La intensificación de las relaciones con la Administración, primera beneficiaria y en la mayoría de los casos promotora principal de los procesos de catalogación.

- Asimismo, es preciso alcanzar una estabilización de las dotaciones presupuestarias que permita poder continuar este proceso y establecer una correspondencia cada vez más estrecha entre el material alojado y el material catalogado e informatizado.

- En tercer lugar, establecer una conexión real entre los distintos centros depositarios de colecciones paleontológicas, a fin de unificar en lo posible los sistemas y programas de bases de datos y de facilitar al máximo el acceso de los investigadores a las colecciones de otros centros.

De esta manera, este tercer factor, la catalogación de las colecciones, garantiza en gran medida los otros dos, de accesibilidad y seguridad. Por último, los centros que mantienen colecciones adecuadamente informatizadas y catalogadas deben ser conscientes de la necesidad de promover e incrementar los fondos bibliográficos correspondientes (o de establecer la posibilidad de acceso rápido a ellos) y de facilitar la realización de réplicas de las piezas en el caso de ser requeridas por los investigadores o por otras instituciones.

\section{Hacia un entendimiento con la Administración}

Urge por tanto progresar en un conjunto de tareas:
- Por un lado, de cara a las administraciones autonómicas, la simplificación de los trámites para la concesión de permisos cuando el equipo solicitante ha realizado ya una solicitud ante la administración al obtener el proyecto de investigación y en el proceso de renovación anual.

- Por otro lado, de cara a la comunidad paleontológica, profundizar en la consecución de un buen entendimiento con la administración sobre el depósito final del material y su accesibilidad, así como sus condiciones de seguridad y catalogación (informatización de las colecciones).

- Por último, si las medidas políticas de regulación, protección y gestión del patrimonio paleontológico son efectivas de cara a la población y al mundo profesional (la comunidad paleontológica), deben serlo igual para los colectivos de aficionados y coleccionistas ilegales. Es urgente, por tanto, el desarrollo de medidas activas y eficaces de vigilancia y acción sobre los puntos protegidos y los yacimientos paleontológicos, al objeto de evitar el expolio, la recolección no autorizada y la destrucción de aquéllos. Se trata de evitar la mala impresión por parte de los paleontólogos profesionales de que la ley únicamente persigue a los que la siguen y la cumplen. Esta situación se ha producido con más frecuencia de lo deseable: los paleontólogos encuentran grandes trabas para realizar su trabajo mientras se continúa produciendo el expolio y la destrucción, en muchos casos impune, de valiosos yacimientos por aficionados y expoliadores profesionales con fines comerciales.

\section{MEDIDAS EDUCATIVO-DIDÁCTICAS}

Si las medidas políticas son importantes para la protección del patrimonio, las medidas educativas no lo son menos. El patrimonio paleontológico es asumido cada vez más en la sociedad como un bien común y como un valor activo de la sociedad, de enorme potencial didáctico, cultural y económico (fundamentalmente, aunque no únicamente, por su importancia turística). Más allá de su importancia científica, el patrimonio paleontológico se proyecta en la sociedad actual sobre campos tan diversos como el cinematográfico, el turístico (como componente de la planificación del ocio), el museístico y didáctico y el puramente patrimonial. Es innegable que la proliferación de manifestaciones culturales, cinematográficas y otras, ha creado una amplia afición en la población por la paleontología. La creación de parques temáticos de contenido paleontológico (el proyecto Dinópolis, gestionado por la Fundación "Conjunto Paleontológico de Teruel" es una buena muestra) refleja esta creciente afición y permite reconocer el patrimonio paleontológico como un valor de potencial económico y cultural innegable.

\section{La concienciación social a través de la gestión del patrimonio}

La gestión social del patrimonio paleontológico, esto 
es, su difusión en la población y su utilización como un instrumento generador de cultura, constituye un proceso que puede y debe ir paralelo al de su protección. Dicho proceso no puede ni debe ignorar el factor local, rara vez tenido en cuenta en el pasado pero actualmente en auge creciente: una pieza o una colección paleontológica valiosa es, cada vez más, sentida como propia por la población de donde procede, y la administración y los paleontólogos deben ser sensibles ante este hecho, propiciando medidas y arbitrando políticas de desarrollo y gestión local del patrimonio.

Un yacimiento de gran importancia en las proximidades de una población puede constituir un lugar tradicional de excursión, recolección incontrolada de fósiles y deterioro progresivo, o también puede convertirse en un importante foco de atracción cultural para la población y el visitante, designado como lugar protegido y fuente de ingresos alrededor del cual se puede desarrollar una infraestructura turística y cultural. En este campo es preciso comprender que una política de orientación didáctica y cultural adecuada puede convertirse en el instrumento más valioso y eficaz de protección del patrimonio paleontológico. En efecto, la implicación de la población en la protección de un patrimonio que se encuentra en su término municipal puede constituir el método más seguro y eficaz de asegurar su protección y su mantenimiento. Esto implicará, lógicamente, desarrollar una política de entendimiento con las autoridades municipales y con las asociaciones culturales locales que van proliferando en la geografía nacional.

\section{Modelos de actuación}

En las comunidades autónomas de La Rioja y Aragón, se encuentran en la actualidad buenos ejemplos de yacimientos que, por su interés paleontológico, han sido acondicionados para su visita y exposición:

(1) Los yacimientos de huellas de dinosaurios de Enciso (La Rioja), han sido objeto de una modélica labor de acondicionamiento. Se cuenta además en esta localidad con un centro de investigación e interpretación. La gestión de este proyecto ha sido llevada a cabo por la Consejería de Obras Públicas del Gobierno de la Rioja, a través de su Comisión de Urbanismo, y por la Consejería de Educación, Cultura, Juventud y Deportes, por medio de su Dirección General de Educación y Cultura. La dirección y supervisión de dicho centro está realizada por investigadores de la Universidad de La Rioja.

(2) El yacimiento del Cámbrico Medio de la Rambla de Valdemiedes, en Murero (provincia de Zaragoza), declarado Bien de Interés Cultural con la denominación de Sitio Histórico, cuenta también con un centro de interpretación. La iniciativa de creación de este Centro partió de investigadores de la Universidad de Zaragoza, y la gestión del proyecto global ha sido realizada por la Diputación General de Aragón (DGA) en colaboración con el Ayuntamiento de esa localidad.

(3) Los yacimientos de mamíferos del Mioceno de Concud (provincia de Teruel), han sido señalizados mediante carteles informativos (y disuasorios), al tiempo que se previene sobre las consecuencias del expolio. Para su promoción y difusión social se ha editado además un conjunto de folletos informativos en los que se explica la formación del yacimiento y su interés patrimonial, la historia de los descubrimientos y la importancia paleontológica de los restos.

(4) En Bueña (provincia de Teruel), se ha llevado a cabo durante los últimos años el acondicionamiento y la instalación de una mesa de interpretación en los yacimientos de pistas fósiles de Megaplanolites ibericus MELÉNDEZ (Calvo et al., 1987) en materiales marinos del Jurásico Superior.

(5) En otros casos, una adecuada gestión ha permitido la creación de museos paleontológicos, como los de Galve y Peñarroya de Tastavins (provincia de Teruel). En ambos casos, ha sido el Gobierno de Aragón, a través de la Dirección General de Patrimonio, la institución que ha financiado estos proyectos, si bien es destacable el papel desempeñado por sus ayuntamientos (al igual que en Bueña), a instancias de los cuales se promovieron estas acciones.

(6) Otro ejemplo destacable lo constituye el Ecomuseo de Molinos (provincia de Teruel) que cuenta entre sus instalaciones con una sala de Paleontología en la que se expone el material paleontológico y arqueológico procedente de las excavaciones de la Cueva de las Graderas. Entre todo el material que allí se recoge, destaca un maxilar inferior de un representante primitivo de Homo sapiens. Esta pieza viene a aumentar el ya destacable valor científico de la colección de este museo (Azanza et al., 1986).

\section{Proyectos culturales y didácticos}

Debe destacarse también los yacimientos que se encuentran en fase de acondicionamiento y protección, como paso previo para su integración en proyectos que contemplen su gestión y promoción social, cultural y didáctica por su interés paleontológico. En este estado, dentro de la Comunidad Autónoma de Aragón, se incluyen:

(1) En Ricla (provincia de Zaragoza), se han dado los primeros pasos hacia la posible creación de un centro de interpretación. El informe previo ha sido llevado acabo por personal investigador de la Universidad de Zaragoza. La actuación incluiría también su integración dentro de un itinerario geológico-turístico y, en última instancia y a iniciativa del Ayuntamiento, la posible creación de un museo paleontológico. Este proyecto está promovido por la Diputación General de Aragón (DGA), a través de la Dirección General de Patrimonio, y a una escala menor por el Ayuntamiento de Ricla con la colaboración de la Asociación Cultural Bajo Jalón.

(2) Igualmente, los yacimientos paleontológicos de vertebrados miocenos de Daroca (provincia de Zaragoza), forman también parte integrante de un proyecto de acondicionamiento y difusión social, promovido y gestionado por la DGA.

(3) El proyecto Dinópolis. Diversos yacimientos paleontológicos de la provincia de Teruel se encuentran aún en proceso de estudio y acondicionamiento para su 


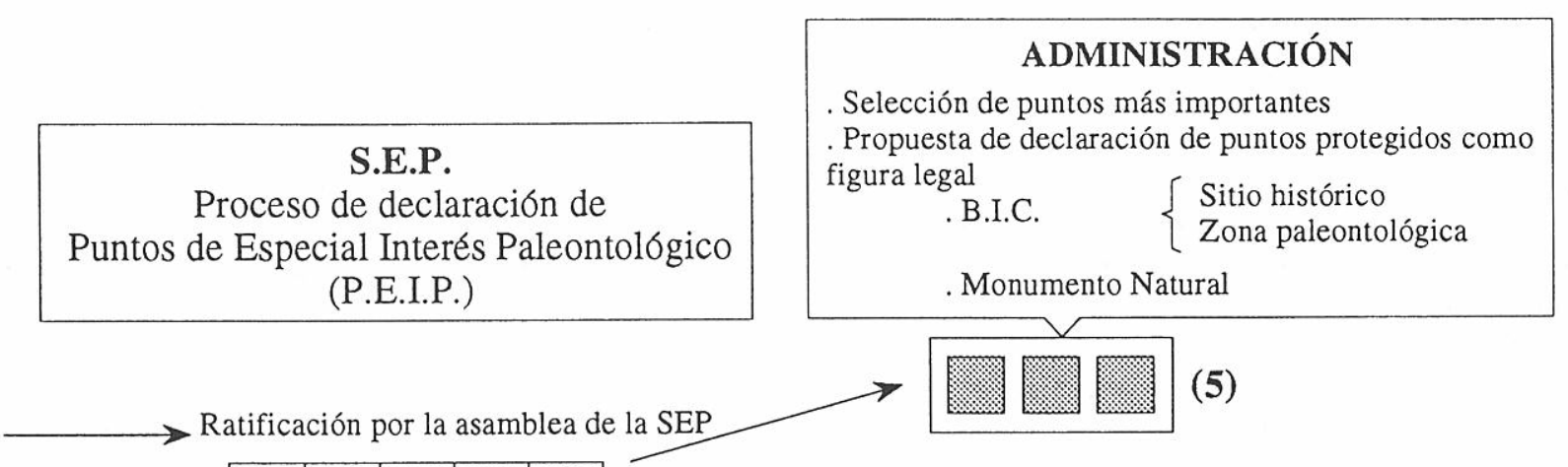

Propuesta declaración de PEIP

(4)

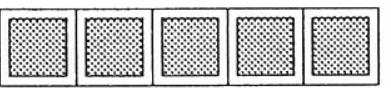

P.E.I.Ps

(Banco de datos paleontológico - SEP)

Estudio y valoración por la C.P.

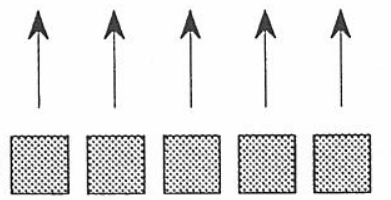

Informe

(3)

Propuesta de PEIP por los investigadores ante la C.P.

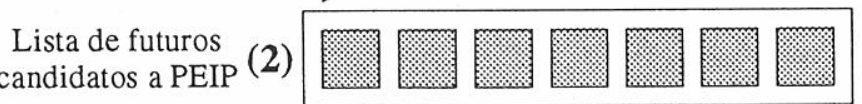

EvalUACIÓN

Yacimientos especialmente $\left\{\begin{array}{l}\text { Riqueza fosilífera } \\ \text { Rareza } \\ \text { Conservación excepcional }\end{array}\right.$ señalados en reuniones o trabajos sobre patrimonio y publicados o inventariados

\section{(1)}

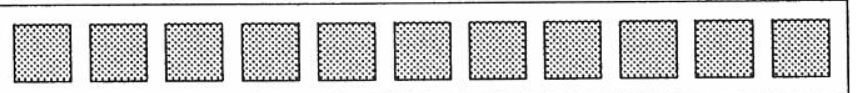

Yacimientos Paleontológicos de una región (conocidos o no)

Figura 6. Proceso de evaluación de los yacimientos paleontológicos y de declaración de Punto de Especial Interés Paleontológico (PEIP) en el interior de la Sociedad Española de Paleontología. Los Yacimientos estudiados y publicados por los investigadores, a través de varios pasos sucesivos, son trasladados a Comisión de Patrimonio de la Sociedad para su análisis patrimonial y tramitación de la propuesta de declaración de PEIP tras su ratificación por la Asamblea anual de la misma. Este conjunto de PEIPs deberá constituir la base de datos sobre la cual la Administración podrá seleccionar a su vez los de mayor interés para su definición como figura legal protegida.

The process of evaluation and nomination of palaeontological site as Point of Special Palaeontological Interest (PEIP) within the Spanish Palaeontological Society (SEP). The process would involve the proposal of studied outcrops, after being published and selected by the researchers, to the Heritage Commission of the Spanish Palaeontological Society for their formal definition as PEIP after being approved by the annual SEP General Meeting. The PEIP list is intended to constitute the primary fossil site database for palaeontologists and the Administration, as regards future definition as legally protection feature.

integración en el proyecto conocido con el nombre de Dinópolis, gestionado por la Fundación Conjunto Paleontológico de Teruel. La gestión y financiación de este proyecto, una combinación de Parque temático de carácter paleontológico y proyecto de difusión turísticocultural de los yacimientos paleontológicos de la provincia de Teruel, corre a cargo del patronato de este proyecto, en el que participan a partes iguales el Gobierno de Aragón, por medio de la Dirección General de Patrimonio, y el Instituto Aragonés de Fomento (IAF), así como otras instancias sociales. La sede central del parque se encontrará en Teruel, en un edificio de nueva planta, en donde las instalaciones se fundirán con atracciones cuyo motivo principal serán reconstrucciones animadas o virtuales de grupos del pasado (invertebrados, reptiles, dinosaurios, mamíferos) representados en el registro fósil de la provincia de Teruel. Este parque temático contará con ramificaciones en lugares donde existen yacimientos paleontológicos de gran tradición o relevancia: Galve, Peñarroya de Tastavins, Concud, Rubielos de Mora y Albarracín.

\section{PAPEL DE LA SOCIEDAD ESPAÑOLA DE PALEONTOLOGÍA}

\section{Órgano interlocutor de la Administración}

La Sociedad Española de Paleontología puede y debe, por medio de sus órganos directivos y representativos y especialmente por medio de la Comisión de Patrimonio, servir de interlocutor entre los paleontólogos y las administraciones, tanto la administración central como las autonómicas, para evitar los conflictos que en ocasiones se han producido, así como para realizar 
labores de asesoramiento en el desarrollo de la legislación sobre patrimonio. A este respecto, en algunas comunidades autónomas, tales como Cataluña, Aragón, Valencia, y en menor medida en La Rioja y Asturias, se han producido sesiones de trabajo o al menos contactos puntuales entre miembros de la Comisión de Patrimonio y las correspondientes direcciones de Patrimonio, con el fin de contribuir a perfilar los criterios básicos en la definición y caracterización del patrimonio paleontológico y su desarrollo legal. Este es un ejemplo de cómo pueden desarrollarse en un futuro las relaciones entre los paleontólogos (la Sociedad Española de Paleontología) y la Administración.

\section{Propuesta de Punto de Especial Interés Paleontológico (PEIP)}

En el seno de la Sociedad Española de Paleontología (SEP), la propuesta de crear una figura de interés patrimonial con objeto de llamar la atención sobre la necesidad de proteger los yacimientos paleontológicos más importantes y más amenazados ha sido uno de los objetivos más debatidos en las reuniones celebradas durante las Jornadas anuales y en las distintas sesiones celebradas sobre patrimonio. Dicha propuesta surgió relativamente pronto en el seno del Grupo de Trabajo sobre Protección de Yacimientos Paleontológicos y se ha ido desarrollando y consolidando desde la constitución formal de la Comisión de Patrimonio (Soria y Meléndez, 1998; Meléndez y Soria, 1994, 1999).

Los Puntos de Especial Interés Paleontológico (PEIP) serían los yacimientos o secciones especialmente señaladas por las características excepcionales de su contenido paleontológico (Fig. 6), sean éstas la riqueza fosilífera, la rareza de los grupos representados, la conservación excepcional de los restos o la importancia biocronológica de las sucesiones registradas. La figura del PEIP se propone como una denominación de carácter interno dentro de la Sociedad Española de Paleontología, sin status legal (Soria y Meléndez, 1993). No obstante, el conjunto de puntos declarados PEIP puede y debe constituir el banco de datos paleontológicos de la Sociedad que, en manos de la Administración, pudiera servir para la posterior declaración de los puntos más relevantes como figura legal protegida.

El proceso de declaración de un yacimiento o una sección como PEIP pasaría por los sucesivos estadios (1) a (4), tal como se muestra en la Fig. 6: en primer lugar, sobre el conjunto de yacimientos paleontológicos de una región, conocidos o no, los paleontólogos, a través de los trabajos monográficos, realizan una primera selección y un inventario de los más relevantes, que constituirá la lista de los futuros candidatos a PEIP. A partir de esta lista, los investigadores pueden elevar una solicitud, acompañada de la ficha correspondiente de cada yacimiento o sección, y un informe para su estudio y valoración por la Comisión de Patrimonio. Tras la evaluación por la Comisión, los puntos propuestos como PEIP son ratificados por la asamblea anual de la Sociedad. De esta manera, la Sociedad Española de Paleontología irá aumentando anualmente el conjunto de puntos considerados como de mayor interés y más necesitados de protección.

A lo largo de las cuatro fases de selección de los PEIP, hasta la declaración posterior de algunos de ellos por la Administración como figura legal protegida (fase $5)$ se produce igualmente una variación en los caracteres tanto científicos como administrativos que contribuyen a otorgarles un mayor valor. Estas variaciones se encuentran representadas en la Fig. 7, en forma de gradientes.

Entre los caracteres que podemos denominar administrativos, en primer lugar el grado de conocimiento social se incrementa desde los primeros estadios pues muchos yacimientos son conocidos por la población antes de ser estudiados por los paleontólogos. El grado de protección es un carácter que debe incrementarse progresivamente, pero no desde los primeros estadios, puesto que muchos yacimientos poco relevantes o en un estado incipiente de estudio no deberían requerir protección ni atención administrativa ninguna. Es este punto el que ha generado numerosas polémicas en la población. De tal manera, el grado o nivel de protección oficial sobre yacimientos o secciones en el escalón inferior debe ser nulo. Por su parte, los riesgos de afección o de deterioro de los yacimientos van en aumento desde el momento en que éstos son conocidos y publicados y, en consecuencia, la vigilancia activa por parte de la Administración debe ir incrementándose en paralelo. No obstante, esta vigilancia debe centrarse en las áreas protegidas (Parques Naturales, etc.) y en aquellos yacimientos declarados como especialmente relevantes (PEIP) o figura legal protegida (Estadios 4 y 5 ).

Entre los caracteres científicos, el grado de conocimiento de los yacimientos por los investigadores aumenta progresivamente desde los primeros estadios, paralelamente a su interés paleontológico. Es obvio que, independientemente de su riqueza o excelencia del afloramiento, el interés paleontológico de un yacimiento es mayor cuanto más conocido es por los paleontólogos y ha sido objeto de más publicaciones científicas, dando origen a listas más amplias de táxones y a la creación de táxones nuevos. Del mismo modo, el interés en los muestreos científicos en un yacimiento o sección estratigráfica aumenta progresivamente de modo similar, paralelamente a la intensidad de su estudio, y a su declaración por los paleontólogos como PEIP o de la Administración como figura legal protegida. Un ejemplo claro lo constituye un afloramiento como el del límite Cretácico-Terciario en Zumaya, cuyo grado de estudio y conocimiento científico es muy alto y el interés de grupos internacionales por realizar muestreos es máximo.

Correlativamente a los caracteres descritos, el grado de accesibilidad al muestreo en las secciones y en los yacimientos paleontológicos debería ser progresivamente restringido al aumentar su interés y su nivel de estudio. De tal manera que los yacimientos en un estado incipiente de estudio o que presentan un escaso o nulo interés científico deberían poder estar abiertos a muestreos o visitas por la población, siempre dentro del 


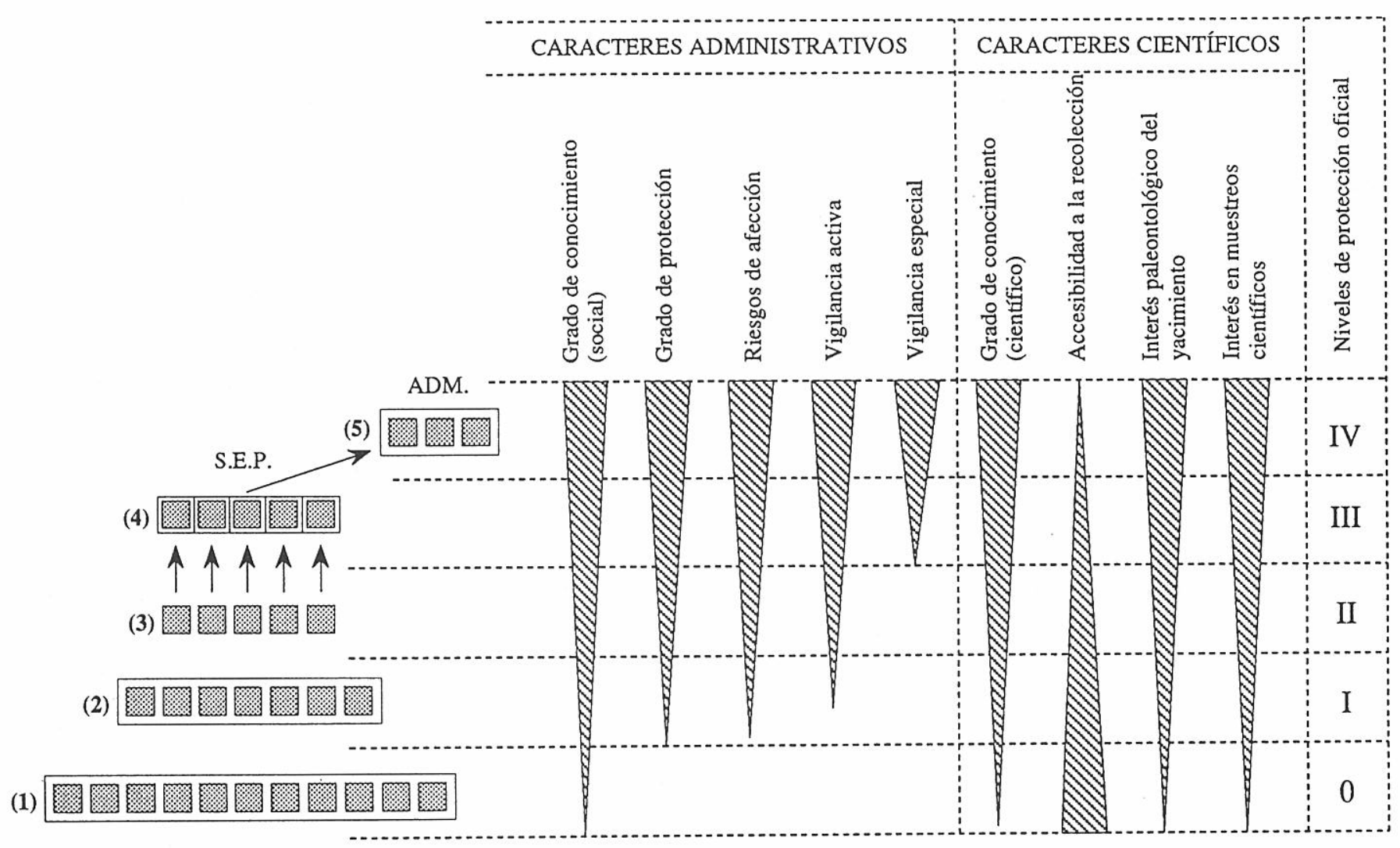

Figura 7. Esquema de la distribución y variación general de los caracteres (administrativos y científicos) que influyen en la valoración, calificación y protección de los yacimientos paleontológicos. Los escalones representados en la parte izquierda se corresponden con los pasos sucesivos propuestos en la Fig. 6.

Distribution and general variation of the main administrative and scientific features affecting the valuation, qualification and protection of palaeontological sites. The different levels on the left correspond to the successive steps proposed in Fig. 6.

marco de la responsabilidad social por el patrimonio paleontológico. Sin embargo, a medida que aumenta el interés o el grado de conocimiento de los yacimientos, parece claro que el acceso a los mismos debe ser progresivamente restringido a los especialistas, y la concesión de permisos por la Administración debe hacerse más estricta.

\section{CONCLUSIONES}

Mientras que la ordenación legal y la gestión política, social, cultural y didáctica del patrimonio paleontológico corresponde a las autoridades políticas, su definición, evaluación y caracterización es una tarea fundamentalmente científica que corresponde a los paleontólogos. De tal manera, en esta labor resulta de primera importancia el desarrollo de una comunicación fluida entre los paleontólogos y la Administración que facilite tanto el asesoramiento sobre las áreas a proteger como la demarcación de criterios sobre la posible creación de nuevas figuras de protección que amplíen el marco legislativo. El ordenamiento legal actual abre un camino para una regulación de las acciones y las excavaciones paleontológicas así como para una gestión, explotación y utilización racional del patrimonio paleontológico. No obstante, para que todo esto sea efectivo y pueda llevarse a cabo de manera fructífera es preciso establecer una relación profunda y una colaboración estrecha entre la comunidad paleontológica y la Administración. Esto implica, por un lado, la simplificación del proceso administrativo que permita a los paleontólogos realizar su trabajo; por otro, la participación de los paleontólogos especialistas en la clasificación, determinación y denominación de los puntos objeto de protección.

La Sociedad Española de Paleontología, como entidad representativa de los paleontólogos españoles, puede desempeñar un importante papel, de coordinación en el interior de la comunidad científica y como organismo intermedio o puente entre los paleontólogos y la Administración. La Comisión de Patrimonio, órgano delegado de la Junta Directiva en este cometido e instrumento de comunicación dentro de la Sociedad, tiene por delante una amplia tarea, tanto de definición del patrimonio y de los criterios de demarcación, como de designación e inventario de los puntos de mayor interés (PEIP) (Fig. 6). Una realización detallada de este inventario deberá permitir además una comunicación más fluida y una mayor capacidad de asesoramiento a la hora de designar los yacimientos que deben ser especialmente protegidos mediante una figura legal específica. Por otro lado, parece también evidente que la Sociedad Española 
de Paleontología tendrá un mayor peso específico y será reconocida como interlocutora de la Administración en la medida en que la Comisión de Patrimonio logre estrechar vínculos con aquélla mediante la creación de una amplia red de miembros corresponsales y colaboradores en las distintas comunidades autónomas. Es fundamental por tanto la participación estrecha y coordinada del mayor número de miembros de la Sociedad en esta tarea.

\section{AGRADECIMIENTOS}

Los autores desean agradecer a los Drs. Jorge Morales y Luis Alcalá (Museo Nacional Ciencias Naturales, Madrid), Sixto Fernández-López (Univ. Complutense, Madrid) y Kevin Page (English Nature, Okehampton, Gran Bretaña) sus numerosas sugerencias y aportaciones, que han contribuido a clarificar muchas ideas expuestas en este texto. Este trabajo se ha realizado en el marco de los proyectos de Patrimonio de la DGA nº: 063/99-A; 063/99-B, y del Instituto Aragonés de Fomento n: 99/0116. Asimismo ha contado con el apoyo económico del Instituto de Estudios Turolenses (CSIC, Teruel).

\section{BIBLIOGRAFÍA}

Alcalá, L. 1992. Mesa redonda: Protección de yacimientos paleontológicos en España. Noticias Paleontológicas, 20, 31-32.

Alcalá, L. and Morales, J. 1994. Towards a definition of the Spanish Palaeontological Heritage. In: Geological and Landscape conservation (Eds. D. O'Halloran, C. Green, M. Harley, M. Stanley and J. Knill). Geological Society, London, 57-61.

Alcalá, L. y Paricio, C. 1984. Protección y conservación de yacimientos paleontológicos españoles. I Congreso Español de Geología, 1, 377-381.

Alcalá, L. y Paricio, C. 1988. Protección y conservación de yacimientos paleontológicos españoles. II Congreso Español de Geología, 1, 253-256.

Andrés-Moreno, J.A. y Royo-Guillén, J.I. 1998. Desarrollo legal del Patrimonio Paleontológico en la Comunidad Autónoma de Aragón. Naturaleza Aragonesa (Sociedad de Amigos del Museo Paleontológico de la Universidad de Zaragoza), 3, 22-29.

Azanza, B., Benito, G., Calvo, J.M., Gil, E., Lorenzo, J.I., Soto, E. y Meléndez, G. 1986. La Cueva de las Graderas, Molinos (Teruel). Instituto de Estudios Turolenses, Teruel, $81 \mathrm{pp}$.

Calvo, J.M., Gil, E. and Meléndez, G. 1987. Megaplanolites ibericus (ichnogen. et ichnosp. nov.) a new trace fossil from the Upper Jurassic (Uppermost Oxfordian) of Bueña (Teruel province, Iberian Chain, Spain). Palaeogeography, Palaeoclimatology, Palaeoecology, 61, 199-204.

Fernández-López, S. 1981. La evolución tafonómica (un planteamiento neodarwinista). Boletín de la Real Sociedad Española de Historia Natural (Sección Geológica), 79, 243-254.
Grupo de Trabajo de Protección de Yacimientos Paleontológicos Españoles, 1993. Mesa redonda sobre Protección de Yacimientos Paleontológicos en España. Málaga, 28 Octubre 1993. Noticias Paleontológicas, 22, 22-24.

López Martínez, N. 1995. Cartas al Editor. Noticias Paleontológicas, 26, 66-68.

Meléndez, G. and Soria, M. 1994. The legal framework and scientific procedure for the protection of palaeontological sites in Spain: Recovery of some special sites affected by human activity in Aragón (Eastern Spain). In: Geological and Landscape conservation (Eds. D. O'Halloran, C. Green, M. Harley, M. Stanley and J. Knill). Geological Society, London, 329-334.

Meléndez, G. y Soria, M. 1997. Problemática actual de la legislación sobre Patrimonio paleontológico en España. Medidas y soluciones. II Reunión Nacional de la Comisión de Patrimonio Geológico, Logroño (1996), 105-110. Zubía, Instituto de Estudios Riojanos, 15, 113120.

Meléndez, G. y Soria, M. 1999. Situación actual del Patrimonio Paleontológico en España. Boletín del Instituto Andaluz de Patrimonio Histórico (IAPH), 29, 128-141.

Meléndez, G., Soria, M. y Martínez-Delclòs, X. 1999. La Comisión de Patrimonio de la Sociedad Española de Paleontología (SEP): Antecedentes, Constitución y Objetivos. Coloquios de Paleontología, 50, 22-36.

Meléndez, G. y Soria-Llop, C. 2000. El Debate sobre el Patrimonio Paleontológico en España: El papel de la Sociedad, las administraciones públicas y los paleontólogos. V Congreso Geológico de España, Alicante. Geotemas, 1(2), 317-324.

Morales, J. 1996. El Patrimonio Paleontológico. Bases para su definición, estado actual y perspectivas futuras. MOPTMA (Madrid): Serie Monografías: El Patrimonio Geológico. Bases para su valoración, protección y utilización, 39-51.

Morales, J., Gómez, E. y Azanza, B. (en prensa). El Patrimonio Paleontológico Español: marco legal, titularidad, gestión y conservación. I Jornadas sobre el Patrimonio de la provincia de Teruel. Rubielos de Mora (Teruel), Septiembre 1998.

Page, K.N., Meléndez, G. and Gonera, M. 1999. Protected sites or protected Heritage? Systems and opinions for palaeontological conservation from a transeuropean perspective. In: Towards the Balanced Managemant and Conservation of the Geological Heritage in the New Millenium (Eds. D. Barettino, M. Vallejo and E. Gallego). Comunicaciones III Simposio Internacional ProGEO (1999), 45- 51.

Sanz, J.L., Buscalioni, A.B., Ortega, F., Pérez, B.P., Moratalla, J. y Raskin, D. 1995. Cartas al Editor. Noticias Paleontológicas, 26, 69-70.

Soria, M. 1993. El Patrimonio Paleontológico de Teruel: Estudio sobre la protección y conservación de yacimientos de especial interés. Instituto de Estudios Turolenses (C.S.I.C.), Teruel.. 145 pp. (Inédito).

Soria M. y Meléndez, G. 1993. La protección de yacimientos paleontológicos en Aragón (España). 
Modelo de actuación sobre protección de yacimientos en las localidades de Ricla y Aguilón (Provincia de Zaragoza, Aragón). Comunicaciones IX Jornadas de Paleontología (Ed. J.M. González Donoso), 79-84.

Soria, M. y Meléndez, G., 1998. La protección del Patrimonio Paleontológico en España: Actuación de la
Comisión de Patrimonio de la Sociedad Española de Paleontología (SEP). Comunicación, I Sesión Científica: Evaluación y Gestión del Patrimonio Geológico y Minero. Sociedad Española para la Defensa del Patrimonio Geológico y Minero: Sedpgym-Camarasa, 3, 25-33.

Manuscrito recibido: 2 de junio, 2000 Manuscrito aceptado: 17 de julio, 2000 